\title{
La naissance : une continuité au centre de la périnatalogie
}

\section{Birth: continuity in the center of perinatology}

\section{Hernandorena}

(C) Lavoisier SAS 2018

La naissance est par définition au centre de nos pratiques en médecine périnatale.

On peut l'imaginer comme une porte qui s'ouvre sur le chemin de la vie, le long duquel le développement neuropsychologique dépend d'une génétique incontournable et d'un environnement en partie émotionnel.

À la naissance, un enfant vient de parcourir ce chemin au mieux pendant neuf mois, et il va le poursuivre avec les mêmes bases génétiques, mais dans un nouvel environnement extrêmement complexe ayant des liens très forts avec l'anténatal. C'est une continuité. Cet environnement aura encore un impact majeur sur le développement neurologique, cognitif, psychologique. C'est le seul point sur lequel les soignants peuvent influer, aussi bien avant la naissance qu'après.

L'adaptation à la naissance est la première étape concrète ; sa qualité est fondamentale, sa prise en charge est un exemple typique de nos pratiques en périnatalité, c'est-à-dire multidisciplinaire. Des séquelles majeures, aussi bien physiques que psychiques peuvent en découler. C'est la raison pour laquelle le comité éditorial a souhaité monter un dossier sur les actualités en matière de soins en salle de naissance. Il a été coordonné par une équipe de très grande expérience venant d'horizons différents : Claire Boithias du CHU de Kremlin-Bicêtre, Jean François Tolsa du CHU de Lausanne et Jean-Louis Chabernaud du CHU de Clamart.

Depuis quelques années, les soignants ont compris que l'enfant doit être accueilli à la naissance, en privilégiant au maximum la continuité transnatale, donc par les deux parents, plus ou moins aidés par les soignants.

Une transition, notamment respiratoire et circulatoire, se met en place, la pesanteur s'installe, l'environnement est radicalement modifié, mais dans l'ensemble, ce n'est qu'une continuité, avant et après la naissance, il s'agit du même chemin, en particulier sur le plan émotionnel; et il nous faut l'aménager le plus harmonieusement possible.

Aussi, les assistances techniques mises en place en cas de nécessité ne doivent que faciliter, accompagner ce passage et ne pas agresser, y compris quand une aide respiratoire est indispensable. Tous les détails vont compter dans cet objectif de continuité, d'harmonie, en particulier pour les plus faibles. Mais pas que pour ceux-là, car un enfant né à terme au décours d'une grossesse et d'un accouchement normal doit pouvoir bénéficier de mesures visant à adoucir cette étape de transition : il ne doit subir aucun geste brutal ni bien sûr douloureux, et entrer en contact corporel immédiat avec les parents, pour partager les émotions avec eux, retrouver les odeurs, ressentir le toucher, atténuer l'inconfort de la pesanteur et des différentes stimulations sensorielles.

Les sujets qui sont abordés dans ce dossier sont des mises au point de techniques récentes de prise en charge. N'oublions pas de les inscrire dans cette réflexion plus globale sur ce passage du chemin que parcourt le petit être humain, passage au cours duquel la femme et l'homme acquièrent aux yeux du monde le statut de mère et de père avec toutes les émotions attenantes qui doivent être privilégiées et protégées.

X. Hernandorena $(\bowtie)$

1780 route de l'Aqueduc, 13990 Fontvieille, France

e-mail : xavier.hernandorena@orange.fr 